\title{
ER@CEBAF: A test of 5-pass energy recovery at CEBAF
}

\author{
S.A. Bogacz, D. Douglas, C. Dubbe, A. Hutton, T. Michalski, \\ F. Pilat, Y. Roblin, T. Satogata, M. Spata, C. Tennant, M. Tiefenback \\ Jefferson Lab, Newport News, VA 23606, USA
}

I. Ben-Zvi, Y. Hao, P. Korysko, C. Liu, F. Meot, M. Minty, V. Ptitsyn, G. Robert-Demolaize, T. Roser, P. Thieberger, N. Tsoupas Brookhaven National Laboratory

\section{Collider-Accelerator Department Brookhaven National Laboratory Upton, NY 11973}

\author{
U.S. Department of Energy \\ Office of Science, Office of Nuclear Physics
}

Notice: This document has been authorized by employees of Brookhaven Science Associates, LLC under Contract No. DE-SC0012704 with the U.S. Department of Energy. The United States Government retains a nonexclusive, paid-up, irrevocable, world-wide license to publish or reproduce the published form of this document, or allow others to do so, for United States Government purposes. 


\title{
ER@CEBAF: A Test of 5-Pass Energy Recovery at CEBAF
}

\author{
S.A. Bogacz, D. Douglas, C. Dubbe, A. Hutton, T. Michalski, \\ F. Pilat, Y. Roblin, T. Satogata , M. Spata, C. Tennant, M. Tiefenback \\ Jefferson Lab, Newport News, VA 23606, USA \\ I. Ben-Zvi, Y. Hao, P. Korysko, C. Liu, F. Méot, M. Minty, \\ V. Ptitsyn, G. Robert-Demolaize, T. Roser, P. Thieberger, N. Tsoupas \\ Brookhaven National Laboratory, Upton, NY 11973, USA \\ * Co-spokesperson
}

June 6, 2016

\begin{abstract}
Jefferson Lab personnel have broad expertise in the design, commissioning, and operation of multiple energy recovery linacs (ERLs): the CEBAF Front-End Test (early 1990s) [1], CEBAF-ER $(2003)[2,3]$, the IR Free Electron Laser (FEL) Demo, the IR FEL Upgrade, and the UV FEL Driver (1997-2014). Continued development of this core competency has led to this collaborative proposal to explore the forefronts of ERL technology at high energy in a unique expansion of CEBAF capability to a 5-pass ERL with negligible switchover time and programmatic impact to the CEBAF physics program. Such a capability would enable world-class studies of open issues in high-energy ERL beam dynamics that are relevant to future facilities such as electron-ion colliders (EICs) [4, 5, 6, 7]. This proposal requests support from the CEBAF Program Advisory Committee to seek funding for hardware installation, and a prospective 12 days of beam time circa Fall 2018 for commissioning this high-energy multi-pass ERL experiment in CEBAF.
\end{abstract}

\section{Contents}

1 Executive summary $r$

2 Introduction and Overview $\quad 3$

3 Hardware and Engineering Layout $\quad \mathbf{8}$

3.1 New Pathlength Chicane . . . . . . . . . . . . . . . . . . . . . . . . . 8

3.2 New Extraction Region and Beam Dump . . . . . . . . . . . . . . . . . . . . 9

3.3 Magnet Specifications . . . . . . . . . . . . . . . . . . . . . . . . . . . . 11

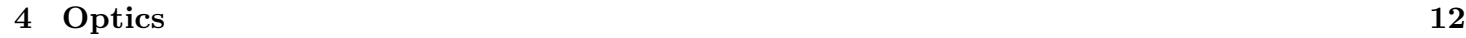

4.1 Longitudinal Match and Considerations . . . . . . . . . . . . . . . . . . . . 12

4.2 Transverse Match and Considerations . . . . . . . . . . . . . . . . . . . . . 15

5 Diagnostics and Measurements $r$

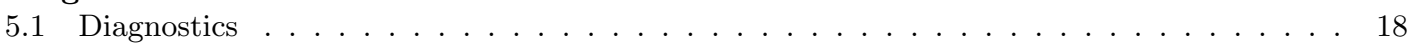

5.2 Measurements . . . . . . . . . . . . . . . . . . . . . . 20

6 Cost and Schedule $\quad 21$

6.1 Installation Cost and Schedule . . . . . . . . . . . . . . . . . . . . . . . . 21

6.2 Staged Commissioning Schedule . . . . . . . . . . . . . . . . . . . . 22 


\section{Executive summary}

The use of energy-recovery linac (ERL) accelerator technology to provide improved beam quality and higher brightness continues to be the subject of active community interest $[8,9]$, and active accelerator development including potential electron-ion colliders (EICs) [4, 5, 6, 7]. Jefferson Lab has been a leader in ERL technology and experiment for over 20 years.

Jefferson Lab experience includes the CEBAF-ER experiment in 2003 [2, 3], which was the first demonstration of high-energy, large-scale superconducting RF energy recovery. Though ongoing projects continue to be proposed using even higher energy ERL parameters, and challenges of high energy ERLs were documented in 2003 [10], the 2003 CEBAF-ER experiment continues to hold the record for high energy ERL demonstration.

With the completion of the CEBAF $12 \mathrm{GeV}$ upgrade, we are now poised to add multi-GeV ERL capability to CEBAF [11]. ERLs in the multi-GeV range rely on efficient recirculation and energy recovery of electron beams that are disrupted by, and lose substantial energy to, incoherent synchrotron radiation (ISR). This is a new ERL regime that has not been experimentally explored. The CEBAF $12 \mathrm{GeV}$ accelerator is the only existing facility that can study ERL dynamics in this regime.

We have designed a new pathlength chicane and low-energy extraction line that enable studies of 1-pass and 5-pass high energy ERL dynamics with negligible switchover time and programmatic impact to the CEBAF physics program. These installations, described in Section 3, require no new magnet design, are designed to remain in place permanently, and do not interfere with any capability of routine CEBAF $12 \mathrm{GeV}$ operations.

Investigations of longitudinal and transverse accelerator optics described in Section 4 indicate that 5-pass energy recovery experiments in ER@CEBAF are feasible with this configuration, up to an energy gain/linac pass of $700 \mathrm{MeV}$, with a possible stretch to $750 \mathrm{MeV} /$ linac pass. This is constrained by understood momentum aperture in the lowest-energy arcs, the maximum energy difference between accelerated and decelerated beams due to ISR, and the capability in CEBAF to perform a longitudinal match.

Section 5 describes additional instrumentation and beam measurements required to commission 5-pass energy recovery. This includes the use of existing RF separators to extract beam on decelerating passes to existing extraction lines for decelerating beam characterization. We expect that initial commissioning will follow a path similar to the 2003 CEBAF-ER experiment:

- Commission dump line/diagnostics (NL acceleration, SL deceleration)

- Commission pathlength chicane and 1-pass ERL

- Commission 5-pass ERL (with incremental beam diagnostics as described in Section 5)

The anticipated costs for new equipment acquisition and installation, and an installation schedule, are outlined in Section 6. Costs including oversight and contingency are approximately $\$ 1.03 \mathrm{M}$ with 6 months of design/fabrication and 4 months for installation and checkout, consistent with a typical CEBAF summer SAD.

This joint proposal by Jefferson Lab and BNL collaborators requests support from the CEBAF Program Advisory Committee to seek funding for hardware design and installation, and a prospective 12 days of beam time circa Fall 2018 for commissioning this high-energy multi-pass ERL capability in CEBAF, and initial beam studies. We anticipate that commissioning of this capability will enable later ERL beam studies consistent with routine beam studies schedules within the CEBAF $12 \mathrm{GeV}$ program. 


\section{Introduction and Overview}

Energy recovery linacs (ERLs [10]) accelerate electron bunches of linac quality, and then recover beam energy by deceleration through the same linac, before dumping the bunches at low (injection) energy. Energy recovery has the benefits of supporting high beam energy and power while maintaining high beam quality, including small beam sizes as delivered by linacs; minimizing activation by dumping low-energy (and thus low-power) beam; and ensuring power-efficient accelerator operation.

The landscape of past, existing, and planned ERL projects is shown in Fig. 1 and Table 1. The 2003 CEBAF-ER one-pass ERL demonstration $[2,12,10]$ of just over $1 \mathrm{GeV}$ remains the highest-energy ERL demonstration on record, and the highest superconducting RF (SRF) ERL demonstration on record by nearly an order of magnitude. Several planned ERL projects, including electron-ion collider (EIC) projects $[6,7,4]$, propose use of ERLs 1-2 orders of magnitude higher in energy than the 2003 CEBAF-ER demonstration.

The upgraded CEBAF accelerator is the only facility worldwide to have the energy reach to demonstrate ERL performance in the multi-GeV range, and ERL performance in a large-scale SRF facility. Enabling this expansion of CEBAF $12 \mathrm{GeV}$ capability to include multipass energy recovery, ER@CEBAF, and study of this capability with beam, are the subjects of this proposal.

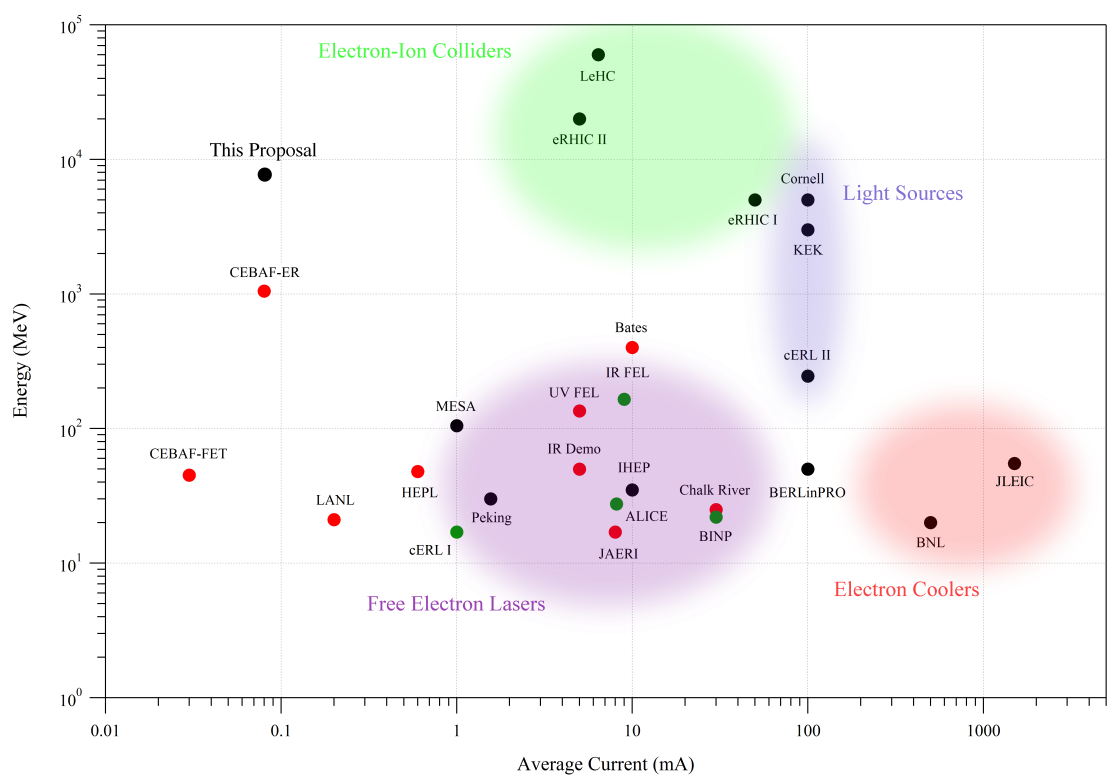

Figure 1: The current ERL landscape: red markers denote previous ERL demonstrations, green markers indicate current ERLs, and black markers represent future ERLs. This proposal is indicated in the upper left. [13]

Table 1: Parameters of Some Past and Present ERLs

\begin{tabular}{lccccc}
\hline Facilities & $\begin{array}{c}\text { CEBAF } \\
\text {-ER }\end{array}$ & $\begin{array}{c}\text { JLab } \\
\text { IR-FEL }\end{array}$ & $\begin{array}{c}\text { JLab } \\
\text { UV-FEL }\end{array}$ & BINP & $\begin{array}{c}\text { KEK } \\
\text { cERL-I }\end{array}$ \\
\hline Linac E $[\mathrm{MeV}]$ & 1050 & 165 & 135 & 10 & 17 \\
Num. of passes & 1 & 1 & 1 & 4 & 1 \\
Current $[\mathrm{mA}]$ & 0.08 & 9 & 2.5 & 30 & 1 \\
\hline
\end{tabular}




\section{Motivation for ERL R\&D at CEBAF}

Development of ERL technology requires that several challenges be met, as outlined in [8, 10, 9, 14]. These include proper design of multi-pass optics, fine control of beam stability and losses (halo), preservation of $6 \mathrm{D}$ bunch quality, polarization (on the accelerated phase), energy recovery efficiency, multiple-beam diagnostic devices, and development of ERL-specific commissioning and optics tuning procedures.

Scaling of energy recovery to multi-GeV energies also encounters incoherent synchrotron radiation (ISR) energy loss and spread, which asymmetrize accelerated and decelerated beam energies and profiles. These asymmetries substantially complicate multi-pass energy recovery and matching, and ultimately limit the energy reach of ER@CEBAF due to recirculating arc momentum acceptance (Section 4).

Scaling of energy recovery to a large-scale SRF installation also raises concerns about multi-pass beam breakup (BBU), an instability that has previously been seen in CEBAF [15] and studied in detail in the Jefferson Lab FEL [3]. There are open questions about scaling of instability thresholds to higher beam energies and a large-scale SRF installation [16] that could be addressed at ER@CEBAF.

We propose ER@CEBAF to experimentally address these challenges with unique new additional accelerator capability. Whereas ER@CEBAF enables experimental exploration of multiple-pass, multiGeV ER, other operating installations are limited in number of passes and energy (Table 1).

\section{Energy Recovery at CEBAF and Jefferson Lab}

CEBAF presently accelerates CW beams for delivery to Hall D at $12 \mathrm{GeV}$ (11 linac passes) and Halls A-C at $11 \mathrm{GeV}$ and below (up to 10 linac passes). After use in the halls, beam is delivered to the respective hall beam dumps at beam power up to $1 \mathrm{MW}$ for Halls A and $\mathrm{C}$ (high current halls), $60 \mathrm{~kW}$ for Hall D, and $1.5 \mathrm{~kW}$ for Hall B (low current halls). All beam is accelerated on RF crest and there is no energy recovery in routine CEBAF operations.

Energy recovery would be made feasible in CEBAF by the addition of two modest hardware sections: a pathlength chicane insertion at the start of Arc A, and a low-power dump line at the end of the south linac (SL), before the first west spreader dipole magnet. These areas are indicated in Fig. 2, and their designs are detailed in sections 3.1 and 3.2 of this document respectively. These sections are designed to remain in place permanently, and do not interfere with any capability of routine CEBAF $12 \mathrm{GeV}$ operations.

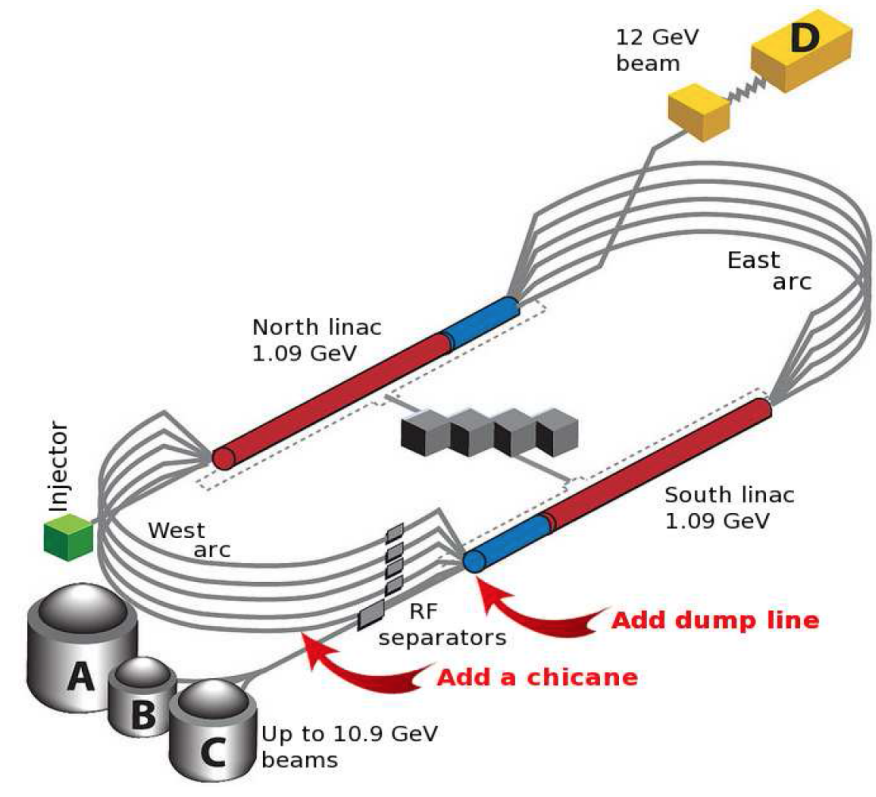

Figure 2: The CEBAF accelerator, with arrows indicating planned hardware installations for the ER@CEBAF experiment. 
Jefferson Lab has broad expertise in the design, commissioning, and operation of multiple ERLs: the CEBAF Front-End Test (early 1990s) [1], CEBAF-ER (2003) [2, 3], the IR Free Electron Laser (FEL) Demo, the IR FEL Upgrade, and the UV FEL Driver (1997-2014). Members of the present collaboration from both Jefferson Lab and BNL are also collaborating on a separate, funded ERL study (C $\beta$ at Cornell) to explore FFAG technology for large momentum aperture recirculation arcs [17]. C $\beta$ and ER@CEBAF complement each other in the ERL landscape and address different ERL questions, but both are relevant to future accelerators. The present collaboration maintains world-leading expertise in ERL design and dynamics considerations necessary to successfully commission the proposed ER@CEBAF experiment.

The 2003 CEBAF-ER experiment, of which ER@CEBAF in the present proposal can be seen as an extension, demonstrated high energy, 1-pass up/1-pass down energy recovery. Measurements included transverse emittances and momentum spread of accelerated and ER'ed beams, halo, and RF system response to ER [3]. Multiple-pass ER at 5-10 GeV, with a $20 \mathrm{~mA}$ (100-200 MW) beam, was further contemplated at that time [2].

\section{Electron-Ion Colliders}

An electron-ion collider (EIC) with CM energy 20-100 GeV and electron beam energy of 5-20 GeV is the next high priority large facility in the 2015 DOE NP Long Range Plan (LRP) [18]. The LRP also recommends "vigorous. . . accelerator R\&D in support of. .. the EIC". In that context, BNL collaborators are developing an ERL-ring scheme based on the RHIC collider (Fig. 3), with relevant parameters as listed in Tables 2 and $3[6,19]$.

The proposed ER@CEBAF energy and scale (up to $11 \mathrm{GeV}$ with 10 linac passes up/down) approach the listed eRHIC parameters (up to $20 \mathrm{GeV}$, with 24 linac passes up/down). All aspects of highenergy, multipass, SRF ERLs to be studied at ER@CEBAF are of direct interest to eRHIC collaborators. ER@CEBAF and eRHIC parameters are compared more directly in Table 3.

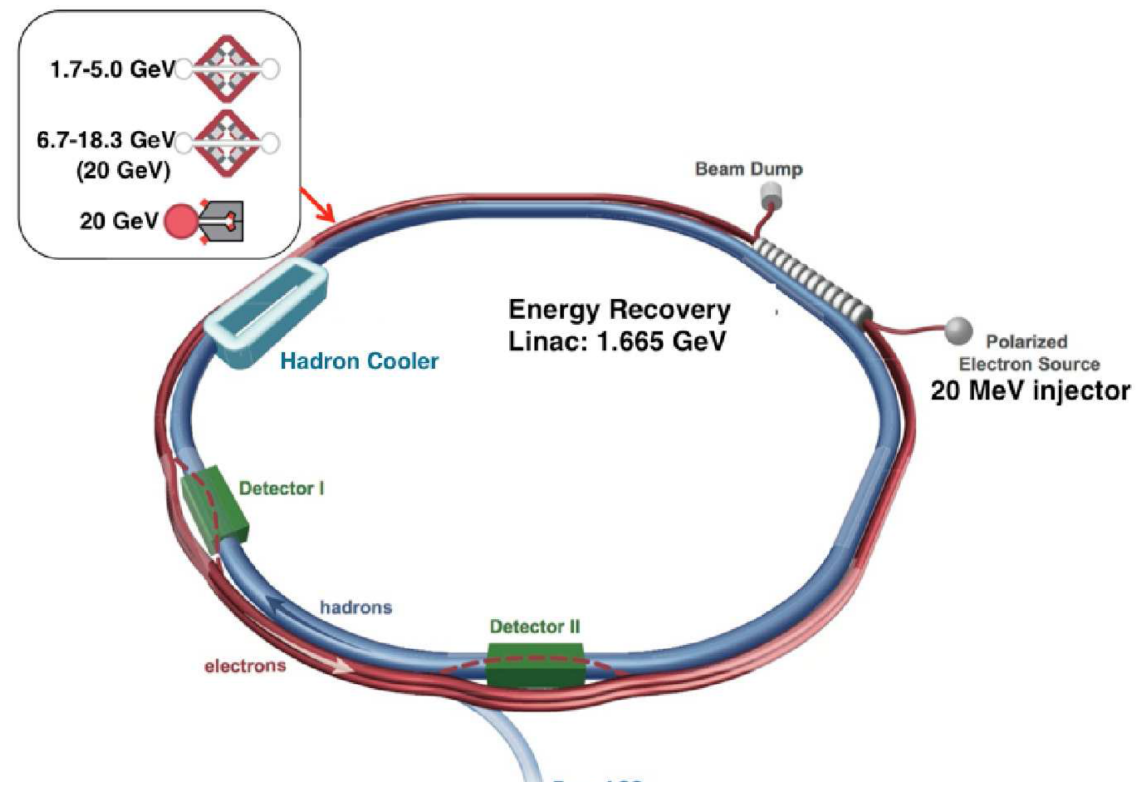

Figure 3: The eRHIC ERL (linac and recirculation loops in red), located in the RHIC tunnel along side the RHIC collider blue ring.

Similar to eRHIC, the linac-ring version of the LHeC EIC at CERN is based on an ERL for the electron beam, and on the existing LHC or a future FCC for the hadron beam [7]. The ERL has a similar layout to CEBAF, yet with parameters in much higher range: two $10 \mathrm{GeV} \mathrm{SC}$ linacs, $500 \mathrm{MeV}$ injection, 6 recirculation arcs, $9 \mathrm{~km}$ circumference, and $400 \mathrm{MW}$ beam power. The proposed LHeC ERL faces even greater challenges towards efficient beam handling and energy recovery than ER@CEBAF 
Table 2: Selected BNL eRHIC EIC Beam Parameters

\begin{tabular}{lcccc}
\hline Species & $\mathrm{e}$ & $\mathrm{p}$ & ${ }^{3} \mathrm{He}$ & $\mathrm{A}$ \\
Energy, max. [GeV, GeV/u] & 20 & 250 & 167 & 100 \\
Beam current, max. [mA] & 50 & 400 & \multicolumn{2}{c}{200} \\
Polarization [\%] & 80 & 70 & 70 & - \\
\hline
\end{tabular}

Table 3: CEBAF and eRHIC Parameters. Energy and beam current are flexible, with ultimate limitations: $1 \mathrm{MW}$ beam power in CEBAF, 2.5 MW SR loss in eRHIC.

\begin{tabular}{|c|c|c|}
\hline & CEBAF & eRHIC \\
\hline Energy [GeV] & 12 & $3.3-20$ \\
\hline Beam current, max. $[\mathrm{mA}]$ & 0.1 & $26-50$ \\
\hline SR loss, max. [MW] & 0.005 & 2.5 \\
\hline Numb. of linacs & 2 & 1 \\
\hline Linac energy $[\mathrm{GeV}]$ & 1.09 & 1.67 \\
\hline Linac passes & $\leq 11$ & $\leq 12$ \\
\hline \multicolumn{3}{|l|}{ Bunch Parameters: } \\
\hline Bunch frequency $[\mathrm{MHz}]$ & $31-1497$ & 9.4 \\
\hline ppb, max. $\left[10^{10}\right]$ & $10^{-4}$ & $1.7-3.3$ \\
\hline rms $\epsilon_{x, y, \text { norm. }}$, inj. $[\pi \mu \mathrm{m}]$ & 3 & $10-70$ \\
\hline rms length $[\mathrm{mm}]$ & $0.09-0.15$ & 3 \\
\hline $\operatorname{rms} \Delta E / E$ & $<10^{-4}$ & $<10^{-3}$ \\
\hline \multicolumn{3}{|l|}{ Linac Parameters: } \\
\hline Length $[\mathrm{m}]$ & 250 & 176 \\
\hline RF freq. $[\mathrm{MHz}]$ & 1497 & 647 \\
\hline Numb. of cavities & $2 \times 200$ & 80 \\
\hline Cavities type & 5- \& 7-cell & 5-cell \\
\hline Cavity gradient $[\mathrm{MV} / \mathrm{m}]$ & $7-20$ & 18 \\
\hline
\end{tabular}

or eRHIC. The ER@CEBAF project offers a unique opportunity of early exploration of multiple-pass, multi-GeV parameter range towards these goals. CERN has expressedly manifested its interest for the project $[20,21]$.

\section{Beyond the EIC}

For the very reasons that render it attractive for the EIC — bunch quality and polarization determined by the source, high energy, high current, high duty cycle, energy efficiency, low energy beam dump SRF ERL technology is contemplated in a variety of other applications in place of storage rings or linacs $[10,8]$.

- synchrotron radiation sources in multi-GeV energy range, with beam current $100 \mathrm{~mA}$ and beyond, nanometer emittance, femtosecond bunches, storage ring level of energy efficiency performance

- higher power, short wavelength FELs, with as an example JLab ER-FEL which reached 1.3 MW CW beam power at $150 \mathrm{MeV}$, whereas some FEL projects aim at multi-MW range with $100 \mathrm{~mA}$ level beam current, hundred $\mathrm{MeV}$ energy level

- electron cooling devices, an application where, in addition to high brightness and low energy spread, high bunch charge/high average current are required.

Achieving performances of high bunch brightness and high beam power as foreseen in these applications requires overcoming accelerator physics and technology challenges, covering bunch production, 
acceleration/use/deceleration, ER efficiency, high charge bunch dynamics in SRF systems, beam diagnostics, etc. As discussed earlier in this document, using CEBAF is a unique R\&D opportunity to address these challenges. Among near-term ERL projects, it is the only installation that would allow exploration of multiple passes and multi-GeV ER in an SRF environment.

\section{ER@CEBAF Parameters and Objectives}

The objective of ER@CEBAF is to perform tolerance and commissioning studies regarding 6D bunch phase space preservation for high-energy ER at 1-pass $(700 \mathrm{MeV} /$ linac or beyond, in similar conditions as the 2003 CEBAF-ER experiment) and at 5-pass (700 MeV/linac with stretch goal of $750 \mathrm{MeV} / \mathrm{linac}$ ).

Initial goals are beam setup and complete beam characterization in both configurations. Setup includes exploration of tolerances of the longitudinal match and momentum aperture; these will be of benefit to the existing CEBAF $12 \mathrm{GeV}$ beam program. Characterization includes initial, intermediate, and final beam emittances, energy measurements, evaluation of ISR effects and mitigations, beam breakup measurements, and scaling with energy per linac.

An overview of parameters for ER@CEBAF is provided in Tables 4 and 5.

Table 4: Main Machine/Parameter List (Sections 3-4)

\begin{tabular}{cccl}
\hline Parameter & Value/Range & Units & Description \\
\hline$f_{\mathrm{RF}}$ & 1497.0 & $\mathrm{MHz}$ & Standard CEBAF RF frequency \\
$\lambda_{\mathrm{RF}}$ & 20 & $\mathrm{~cm}$ & Standard CEBAF RF wavelength \\
$E_{\text {linac }}$ & 700.0 & $\mathrm{MeV}$ & Energy gain per linac pass (baseline, Section 4.1) \\
$E_{\text {inj }}$ & 79 & $\mathrm{MeV}$ & Energy of beam from injector $\left(=E_{\text {linac }} * 123 / 1090\right)$ \\
$N_{\text {passes }}$ & 1,5 & - & Number of machine passes before energy recovery \\
$\phi_{\text {FODO,NL }}$ & 60 & degrees & Phase advance/cell, north linac \\
$\phi_{\text {FODO,SL }}$ & 60 & degrees & Phase advance/cell, south linac \\
$M_{56}$ (Arc A) & $80-90$ & $\mathrm{~cm}$ & $M_{56}$ compression of arc A \\
$M_{56}$ (other arcs $)$ & 0 & $\mathrm{~cm}$ & $M_{56}$ compression of other arcs \\
$\theta_{\text {extraction }}$ & 8 & degrees & Extraction angle \\
$P_{\text {dump }}$ & 20 & $\mathrm{~kW}$ & Maximum dump power (CEBAF standard) \\
$\phi_{\text {tol }}$ & 0.25 & degrees & Required pathlength control tolerance \\
\hline
\end{tabular}

Table 5: Beam Parameter List (Section 4)

\begin{tabular}{cccl}
\hline Parameter & Value/Range & Units & Description \\
\hline$f_{\text {beam,CW }}$ & 249.5 & $\mathrm{MHz}$ & Standard CEBAF CW bunch repetition frequency \\
Tune mode duty cycle & $1.5 \%$ & - & Tune mode duty cycle relative to CW \\
$I_{\text {beam,maxCW }}$ & 100 & $\mu \mathrm{A}$ & Maximum CW beam current \\
$q_{\text {bunch,maxCW }}$ & 0.2 & $\mathrm{pC}$ & Bunch charge (at 100 $\mu \mathrm{A} \mathrm{CW})$ \\
$\sigma_{\text {bunch,L }}$ & $90-150$ & $\mu \mathrm{m}$ & Bunch length (high energy) \\
$\sigma_{\text {bunch,t }}$ & $300-500$ & $\mathrm{fs}$ & Bunch length (high energy) \\
$\sigma_{\text {bunch, } \phi}$ & $0.16-0.27$ & $\circ$ & Bunch length (high energy) \\
$\varepsilon_{\mathrm{x}, \mathrm{y}, \text { geom,inj }}$ & $10^{-8}$ & m-rad & Transverse RMS geometric emittance at injector \\
$d p / p_{\text {inj }}$ & $<10^{-4}$ & - & Momentum/energy spread at injector \\
$\varepsilon_{\mathrm{x}, \mathrm{y}, \text { geom,extraction }}$ & $\mathrm{o}\left(10^{-8}\right)$ & m-rad & Transverse RMS geometric emittance at 10-pass extraction \\
$d p / p_{\text {extraction }}$ & $2-3 \%$ & - & Momentum/energy spread at extraction $($ ER@CEBAF) \\
\hline
\end{tabular}




\section{Hardware and Engineering Layout}

ER@CEBAF requires two modifications of the existing $12 \mathrm{GeV}$ CEBAF accelerator: a new pathlength chicane in the $5^{\text {th }}$ pass AE region, and a new low-energy extraction region and beam dump extending out of the beamline section between the south linac and start of the west spreader.

\subsection{New Pathlength Chicane}

To invoke energy recovery mode for ER@CEBAF, the beam must pass through an additional half of the CEBAF RF wavelength $(10 \mathrm{~cm})$ in the final arc (Arc A). We plan to implement this with a new $31 \mathrm{~m}$ long chicane from AE01 to AE03, in the matching straight just before the arc. The chicane is configured with four standard CEBAF MBA 3-meter 40-turn dipole magnets as illustrated in Fig. 4. The required maximum individual dipole field at peak beam energy of $7073 \mathrm{MeV}$ is $6858 \mathrm{G}$, with magnet current 345A, comfortably within the MBA magnet operating range up to $9550 \mathrm{G}$ at $480 \mathrm{~A}$.

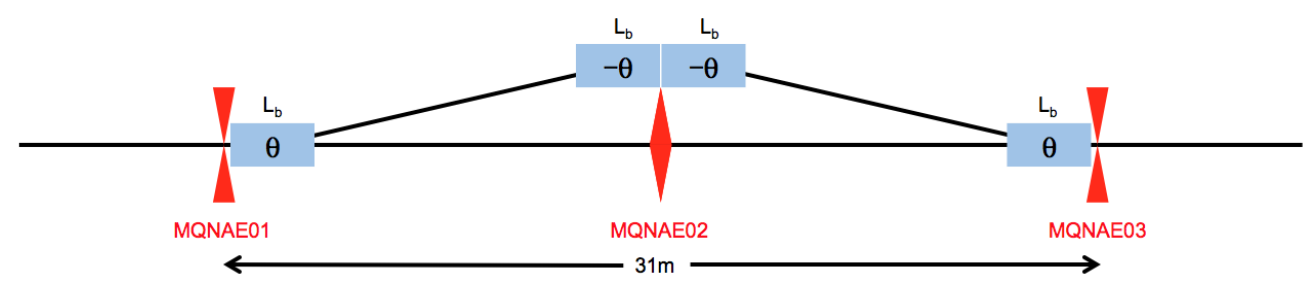

Figure 4: ER@CEBAF new AE region pathlength chicane, with $\theta=5^{\circ}$ and dipole length $L_{0}=3 \mathrm{~m}$.

CEBAF $12 \mathrm{GeV}$ would operate with the chicane dipoles turned off and normal design optics. The chicane would be on during ER@CEBAF studies, bypassing the extraction quadrupole MQNAE02. This configuration requires alternative optics throughout the AE matching section, as illustrated in Fig. 5. These optics provide reasonable beam sizes through the chicane, and match to normal design optics for the A spreader and arc.

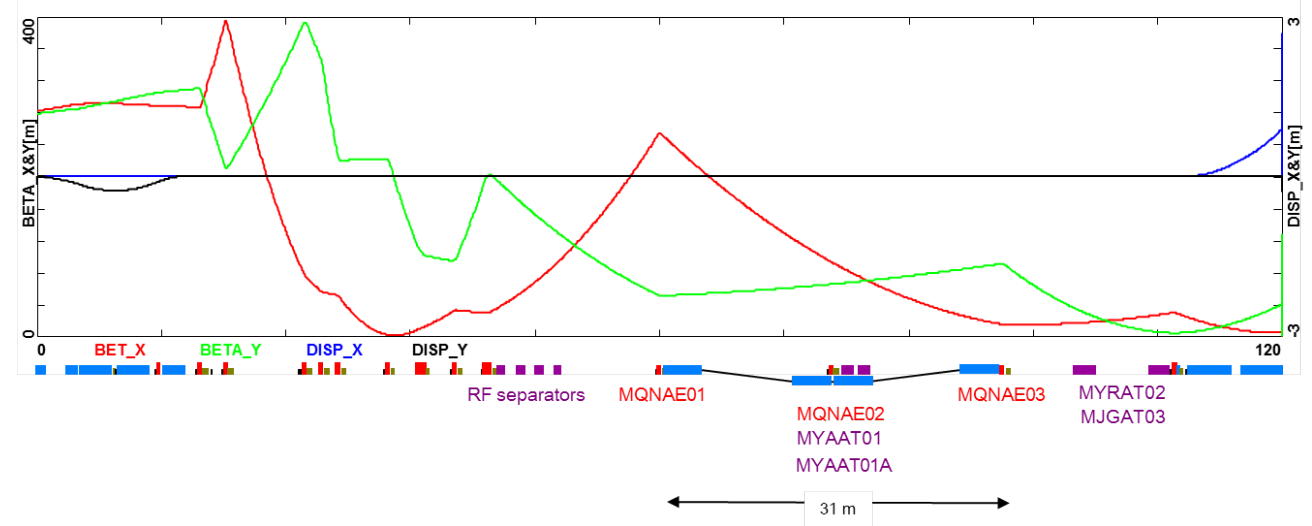

Figure 5: ER@CEBAF pathlength chicane optics, used when chicane is on and bypassing quadrupole MQNAE02.

Support of both "straight through" and "chicane bypass" modes requires a special beampipe design with a $5^{\circ}$ split. This type of vacuum chamber has already been used at CEBAF, e.g. for the Hall A Compton polarimeter chicane [22]. There are no additional vacuum concerns for this insertion.

An engineering perspective layout of the four chicane MBA dipoles, with the middle magnets extending about $1 \mathrm{~m}$ into the aisle and additional lower-energy passes, is illustrated in Fig. 6. This chicane would not interfere with tunnel passage or clearances in this area. 


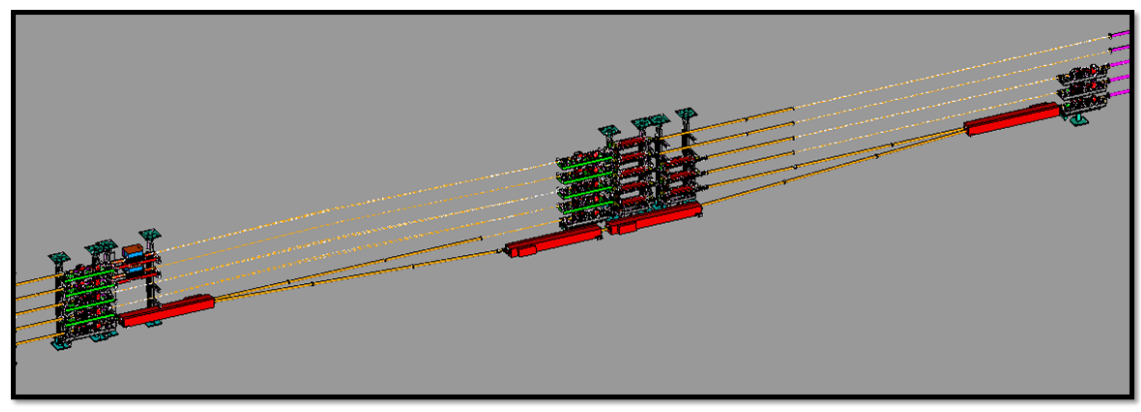

Figure 6: Perspective view of the ER@CEBAF pathlength chicane, showing 2E01-2E03 through AE01-AE03 stacks and magnet stands.

\subsection{New Extraction Region and Beam Dump}

After the last energy-recovery pass through the SL, the ER@CEBAF beam energy is the same as the injector energy, minus small ISR energy losses. Beam up to $\approx 80 \mathrm{MeV}$ (corresponding to the injector energy with $700 \mathrm{MeV}$ /pass linac energy gain, Table 4), will be extracted here and delivered to a diagnostic region and standard CEBAF $20 \mathrm{~kW}$ (low power) beam dump. This rating of beam dump at $80 \mathrm{MeV}$ can handle up to $200 \mu \mathrm{A}$ of $\mathrm{CW}$ beam.

The existing warm space between the (cold) SL26 RF cryomodule and the (warm) first BCOM spreader dipole at the end of the SL is shown in Fig. 7. This area currently contains two girders. The first includes a pump drop, dipole correctors, a BPM, and an MQL quadrupole. The second includes a three-can differential pumping station to provide vacuum isolation between warm and cold regions of the accelerator. A pathlength measurement cavity is also located in this region.

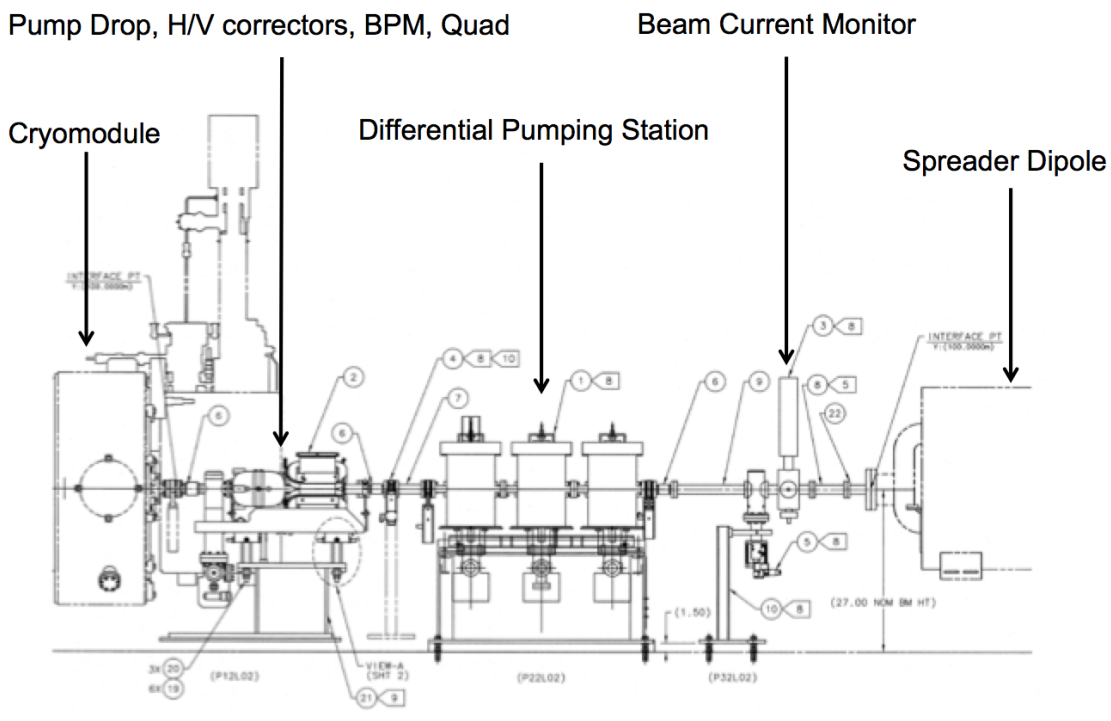

Figure 7: Existing warm space between south linac and start of west spreader.

A reconfiguration of this region to include low-energy extraction capability to a diagnostic and beam dump line is shown in Fig. 8. This includes a standard CEBAF MBL dipole (as used in the injector chicane) for an $8^{\circ}$ bend at the maximum ER@CEBAF injector beam energy of $80 \mathrm{MeV}$, a standard CEBAF $30 \mathrm{~cm} \mathrm{MCA} \mathrm{dipole} \mathrm{magnet} \mathrm{for} \mathrm{bump} \mathrm{closure} \mathrm{(see} \mathrm{below),} \mathrm{a} \mathrm{corrector} \mathrm{dipole/BPM} \mathrm{package,} \mathrm{and}$ relocation of the pathlength measurement cavity and differential pumping station. 


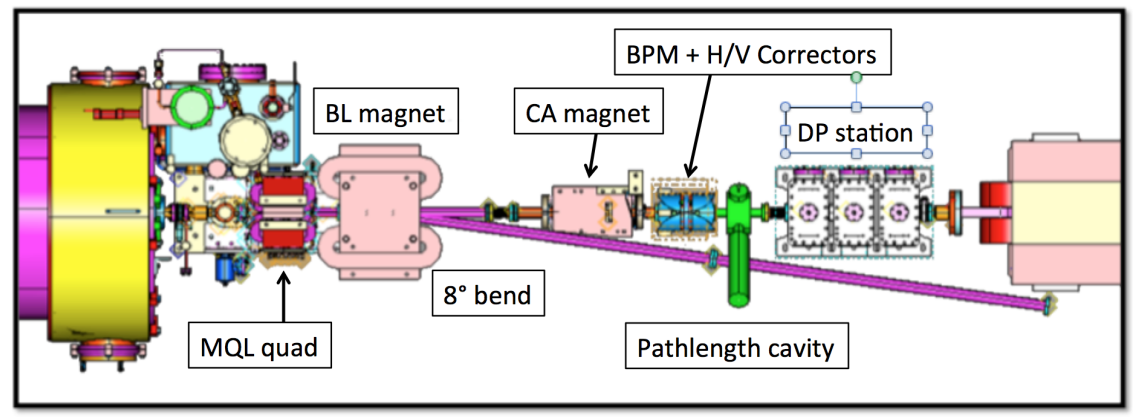

Figure 8: Proposed new warm space between south linac and start of west spreader, including extraction elements for up to $80 \mathrm{MeV}$ ER@CEBAF beam.

At the peak extracted energy of $64.5 \mathrm{MeV}$ (79 MeV injector energy minus $14.5 \mathrm{MeV}$ SR losses), the $30 \mathrm{~cm}$ long $\mathrm{MBL}$ dipole requires $1 \mathrm{kG}$ field $(8.2 \mathrm{~A})$ to bend the beam $8^{\circ}$ for extraction, well within the range of CEBAF standard MBL dipoles.

An important consideration for this design is that up to ten beams (five accelerating and five decelerating) traverse this area, each with different energy. The MBL dipole kicks the lowest energy beam by $8^{\circ}$ as noted above. The next lowest energy beam is $1474 \mathrm{MeV}$ is kicked by $6.1 \mathrm{mrad}$ in the same direction; this stays within the beampipe aperture and is partially compensated by the downstream MCA dipole magnet in this line. Residual orbit displacement for this and higher-energy recirculating beams fits within the first spreader dipole BCOM magnet and other spreader apertures, and is returned to the nominal design orbit by existing orbit correctors in the spreaders.

A perspective drawing of the new dump line is shown in Fig. 9. This dump line includes an additional DP station to isolate the vacuum of the cold SL26 linac module from the warm dump region. It then extends out into the west spreader aisle, and bends parallel to the west spreader with a symmetric BL dipole. A quadrupole girder with BPM, steering magnets, and a standard CEBAF MQB quadrupole doublet provides necessary focusing for the large energy-recovered beam as well as optics control for transverse emittance measurements. BCM, viewer, and harp diagnostics are located just upstream of the dump face.

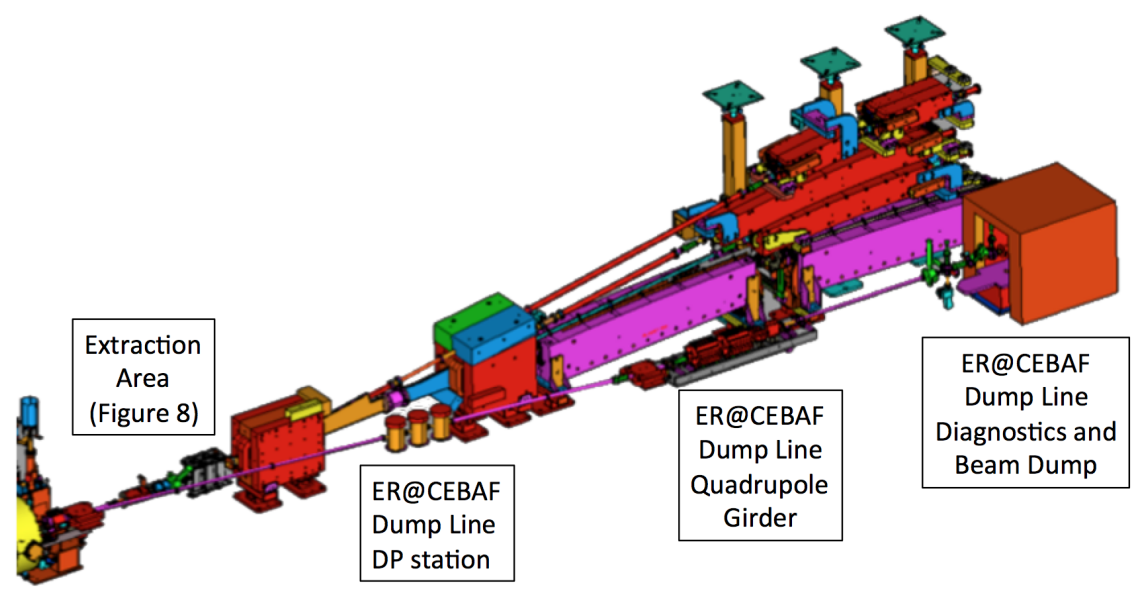

Figure 9: Perspective view of ER@CEBAF dump line in SL spreader region, showing full line from extraction to dump, and nearby SL spreader.

Figure 10 shows the layout of this new dump line in overhead view with more detail, and includes the locations of tunnel walls in this region. Installation of the dump line still provides adequate aisle traffic clearance in this area. 


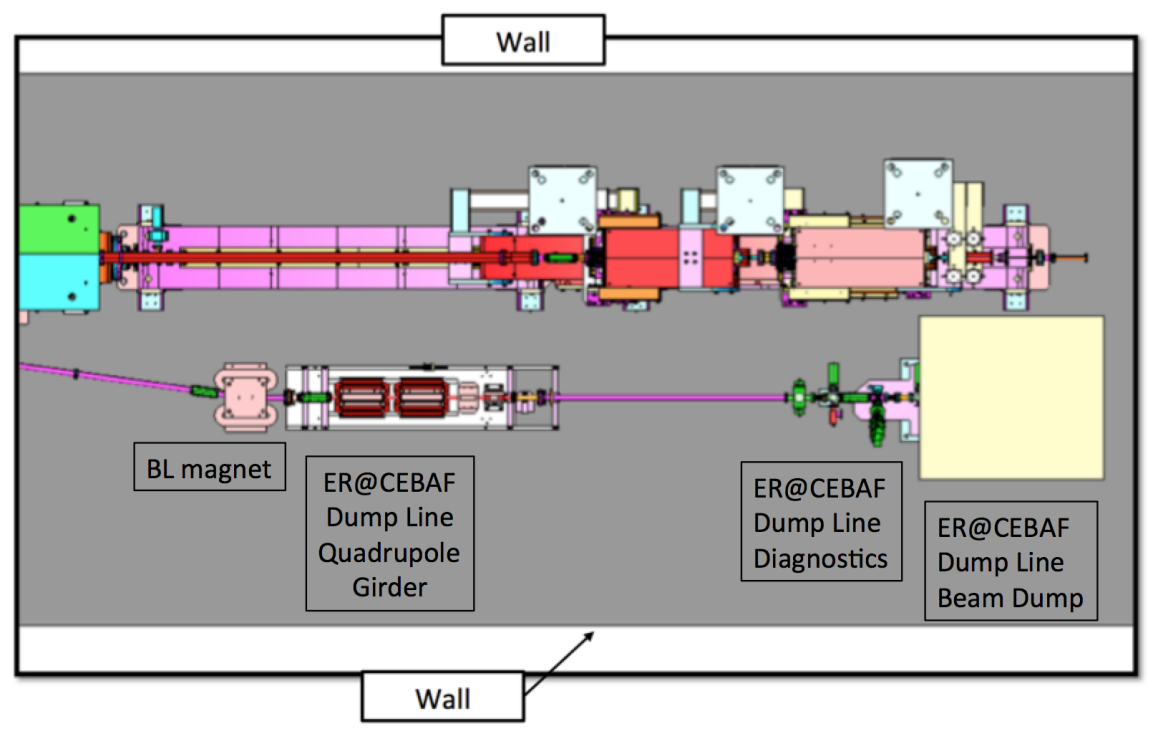

Figure 10: Overhead detail of ER@CEBAF dump line in SL spreader region, showing locations of tunnel walls.

\subsection{Magnet Specifications}

Magnet types and requirements for the pathlength chicane and extraction beamline are summarized in Table 6. All magnets are duplicates of existing CEBAF magnet families. More details are provided in Section 6 on costing.

Table 6: Magnet Types and Parameters for ER@CEBAF Pathlength Chicane and Extraction Beamline: $700 \mathrm{MeV} / \mathrm{linac}$

\begin{tabular}{lccccc}
\hline Area & Type & \# Needed & Length & Field & Current \\
\hline Pathlength chicane & MBA & 4 & $3.0 \mathrm{~m}$ & $6858 \mathrm{G}$ & $345 \mathrm{~A}$ \\
Extraction dipole & MBL & 2 & $0.3 \mathrm{~m}$ & $1000 \mathrm{G}$ & $8.2 \mathrm{~A}$ \\
Extraction dipole & MCA & 1 & $0.3 \mathrm{~m}$ & $1050 \mathrm{G}$ & $10.0 \mathrm{~A}$ \\
Dump quadrupole & MQB & 2 & $0.15 \mathrm{~m}$ & $14 \mathrm{kG}$ & $1050 \mathrm{G}$ \\
\hline
\end{tabular}




\section{Optics}

The optics for ER systems in general, and ER@CEBAF in particular, require careful consideration. These directly address the high-energy ER concerns of phase space matching, phase space preservation, and halo control as outlined in [10]. We address both these areas in the following sections, while noting that establishing beam tuning to experimentally explore these three items in a world-class ER experiment is one of the main objectives of ER@CEBAF.

\subsection{Longitudinal Match and Considerations}

The longitudinal stability in ERLs is dependent upon judicious choices for accelerating and decelerating RF phases, the initial bunchlength and energy spread of the beam, and the chromatic characteristics of the return arcs. ISR-driven energy loss in the high-energy arcs, in both accelerating and decelerating passes, results in two beams of different momentum traversing each arc. Finally, as a consequence of antidamping during deceleration, the relative energy spread of the beam becomes larger during deceleration and energy recovery. All of these effects can be mitigated by performing the appropriate longitudinal phase space manipulations.

The injection chicane, located at the end of the injector, can be used to perform initial bunch compression. We can also separately adjust linac phases for acceleration and deceleration passes, and control $M_{56}$ (linear momentum compaction) in each of the CEBAF arcs separately.

To first order, one must consider limits imposed by the momentum aperture of such arcs, as well as the chromatic effects induced when traversing the quadrupoles. Most CEBAF arcs are equipped with 32 dipoles of 1, 2, 3 or 4 meter length; ARC1 has 16 1m long dipoles, while ARC2 has $162 \mathrm{~m}$ long dipoles. Each arc dipole string is powered by a single power supply, while all CEBAF quadrupoles are individually powered.

\subsubsection{Momentum Acceptance}

The limiting factor for ER@CEBAF with 5 passes is the ARC momentum acceptance, which places a bound on the maximum energy gain one can support in the linacs. Above that energy gain, the ISR losses are sufficiently large that the energy separation between accelerated and decelerated beams in the last arcs is larger than the ARC momentum acceptance.

The standard configuration for the CEBAF ARC1 and ARC2 is high dispersion optics ( 8 and 6 meters peak respectively). For the CEBAF ER experiment, we redesigned these optics to be low dispersion like the other arcs in the nominal CEBAF $12 \mathrm{GeV}$ design to give them larger momentum acceptance. Changing these arcs to this configuration only requires changes to quadrupole magnet setpoints. All arcs are first order (but not second) achromats (Fig. 11). The LiTrack [23] model includes the non-zero second order $T_{566}$ terms that are present in all the return arcs.
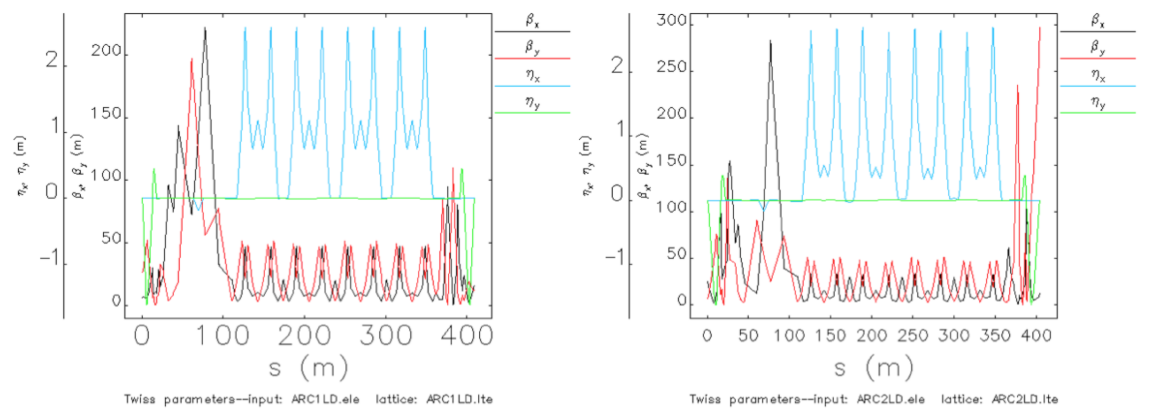

Figure 11: Low dispersion version of Arcs 1 and 2 for the ER@CEBAF experiment.

We performed the initial estimates of the momentum acceptance using a simple spreadsheet and established ISR energy loss and energy spread formulas from Sands [24]. From this we determined 
that the maximum feasible energy gain for ER@CEBAF is $700 \mathrm{MeV} /$ linac, with a likely hard bound somewhere below $750 \mathrm{MeV} /$ linac.

Table 7: Pass-by-pass beam energy parameters for 5-pass ER@CEBAF at 700 $\mathrm{MeV} /$ linac, and phases as described in text. $\Delta E$ is energy lost to $\mathrm{SR}$ in each arc. All energies are in $\mathrm{MeV}$ according to theory; $p_{\text {exit }}$ is from tracking, and includes small effects including spreader/recombiner and quadrupole SR energy losses.

\begin{tabular}{lccccc}
\hline Arc (accelerating) & $E_{\text {entrance }}$ & $E_{\text {exit }}$ & $\Delta E$ & $E_{\text {loss,total }}$ & $p_{\text {exit }}$ (tracking) \\
\hline 1 & 779.00 & 779.00 & 0.00 & 0.00 & 778.98 \\
2 & 1479.00 & 1478.98 & 0.02 & 0.02 & 1478.96 \\
3 & 2178.98 & 2178.88 & 0.10 & 0.12 & 2178.76 \\
4 & 2878.88 & 2878.73 & 0.15 & 0.27 & 2878.48 \\
5 & 3578.73 & 3578.37 & 0.36 & 0.63 & 3577.79 \\
6 & 4278.37 & 4277.89 & 0.49 & 1.11 & 4276.95 \\
7 & 4977.89 & 4977.00 & 0.89 & 2.00 & 4975.99 \\
8 & 5677.00 & 5675.49 & 1.51 & 3.51 & 5674.55 \\
9 & 6375.49 & 6373.10 & 2.40 & 5.90 & 6372.26 \\
10 & 7073.10 & 7070.37 & 2.72 & 8.63 & 7069.98 \\
\hline Arc (decelerating) & $E_{\text {entrance }}$ & $E_{\text {exit }}$ & $\Delta E$ & $E_{\text {loss,total }}$ & $p_{\text {exit }}($ tracking $)$ \\
\hline 9 & 6371.83 & 6369.44 & 2.39 & 11.02 & 6370.19 \\
8 & 5670.90 & 5669.40 & 1.50 & 12.52 & 5672.07 \\
7 & 4970.85 & 4969.97 & 0.89 & 13.40 & 4966.02 \\
6 & 4271.42 & 4270.94 & 0.48 & 13.88 & 4966.61 \\
5 & 3572.39 & 3572.04 & 0.35 & 14.24 & 3568.62 \\
4 & 2873.50 & 2873.35 & 0.15 & 14.39 & 2871.69 \\
3 & 2174.80 & 2174.71 & 0.10 & 14.48 & 2174.30 \\
2 & 1476.16 & 1476.14 & 0.02 & 14.50 & 1477.65 \\
1 & 777.60 & 777.59 & 0.00 & 14.51 & 780.56 \\
dump & 79.04 & - & 0.00 & 14.51 & 83.50 \\
\hline
\end{tabular}

To maximize the momentum acceptance, we adopted the following method: In each Arc, we calculated the average momentum for the accelerated and decelerated beams and calculated the resulting average momentum. In this study, we chose to ignore the effect of slight mispowerings in these quadrupoles as it is within the domain of accuracy of the absolute quadrupole field (sub percent) and the off momentum optics do not differ significantly in terms of beam envelope. This assumption will be lifted in future simulations.

\subsubsection{Longitudinal tracking}

Next, LiTrack was used to study the longitudinal behavior of ER@CEBAF for $750 \mathrm{MeV} /$ linac. By default, LiTrack does not support ISR modeling. It was "added" by using a fictitious cavity with very low frequency $(1 \mathrm{~Hz})$ set to decelerate by the amount of energy that was lost in the previous arc due to ISR. Likewise, the growth in energy spread due to the ISR was taken into account with the appropriate LiTrack Element after each arc.

The same setup was simulated using ELEGANT [25]. There is good agreement between ELEGANT and LiTrack. Figure 13 shows the two calculations side by side at the ER recovery dump

Slight differences may be accounted for by the fact that the ISR energy loss in quadrupoles is taken into account by ELEGANT and not by LiTrack. Another effect that we get via tracking is the fact that the RF timing depends on the choice of fiducial (head of bunch, centroid or tail).

From these studies, we determined that for a $700 \mathrm{MeV} /$ linac setup with the injector set to accelerate to $79 \mathrm{MeV} / \mathrm{c}$, the $0 \mathrm{L04}$ zone phase offset set to $-12^{\circ}$, the north and south accelerating at $92.2^{\circ}\left(2.2^{\circ}\right.$ 

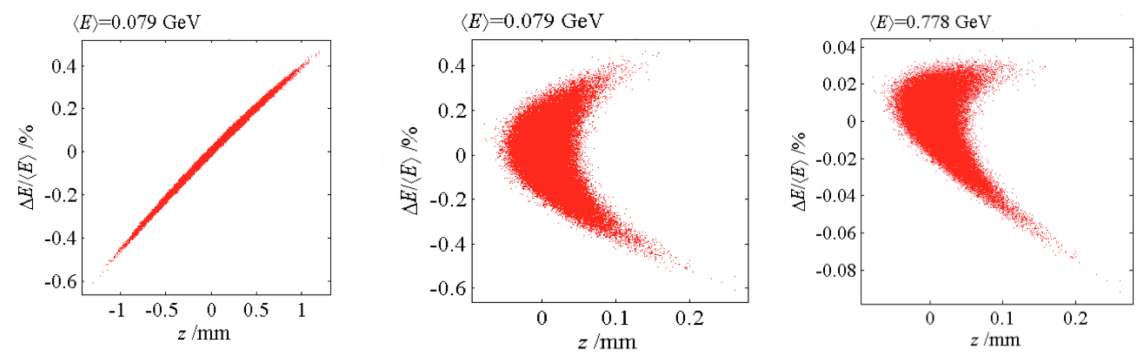

Figure 12: Longitudinal phase space at the entrance and exit of bunch compression injector chicane. No $T_{566}$ correction was used so a curvature remains. The right figure is the phase space at the entrance of ARC1 after traversing the NL.
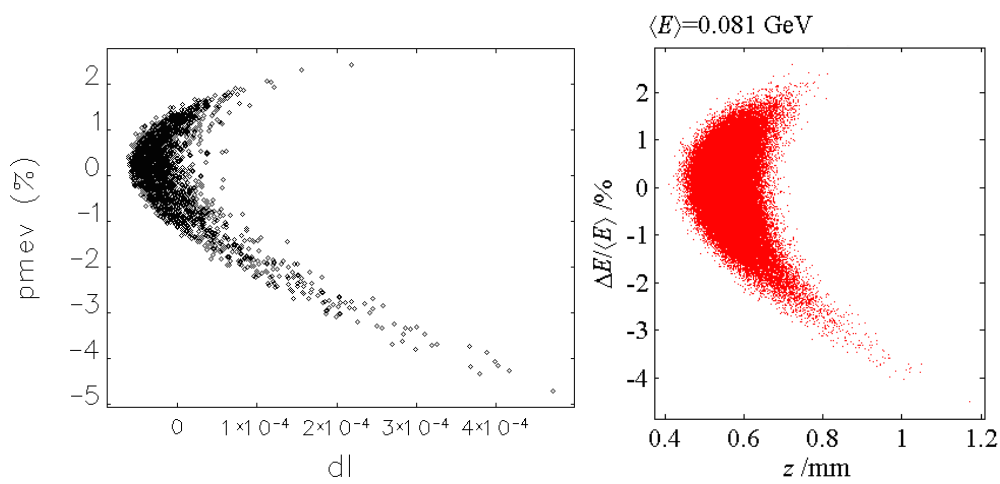

Figure 13: Phase space at the ER@CEBAF dump, for elegant (left) and LiTrack (right).

from crest) and decelerating at $266^{\circ}\left(-4^{\circ}\right.$ from crest) yielding a beam that is energy recovered at 86 $\mathrm{MeV} / \mathrm{c}$ with an energy spread of 2-3\% at the ER dump. It is likely one can optimize this energy spread further by altering the $M_{56}$ in the arcs and the linac phases. This current solution will serve as a starting point for further optimizations. We believe it is good enough to be energy recovered. We note that in order to have a truly optimized ERL one would need to repower sextupoles in the arcs and install sextupoles in the chicane to correct the second order curvature by adjusting the $T_{566}$. However, as demonstrated by tracking, one can still recover the beam in these less than optimal conditions without further modifications to the CEBAF lattice.

\subsubsection{RF Stability Requirements}

Preliminary estimates indicate that the linac RF phase needs to be controlled within a $0.25^{\circ}$ or better. We envision using the MOMOD system $[26,27]$ to lock the linac phases within $0.1^{\circ}$. This is based on existing pathlength hardware and software currently being commissioned for CEBAF $12 \mathrm{GeV}$ operations, and that level of pathlength control will be established during CEBAF beam studies independent of this proposal.

\subsubsection{Field Quality Requirements}

No constraints were placed on the field quality in the magnets besides those imposed by the $12 \mathrm{GeV}$ machine since this is not a green field design but rather an attempt to reuse the existing machine. 


\subsection{Transverse Match and Considerations}

We will introduce optimum design of multi-pass linac optics for a recirculating linac (RLA) based on a large-scale superconducting linac. That strategy was originally used in the design of $60 \mathrm{GeV}, 6$-pass RLA for the LHeC [28]. Here it is extended to 10 passes (5 passes up +5 passes down) for the proposed ER@CEBAF experiment.

\subsubsection{Multi-pass Linac Optics}

Energy recovery in a racetrack topology explicitly requires that both the accelerating and decelerating beams share the individual return arcs. This in turn, imposes specific requirements for TWISS functions at the linacs ends: the TWISS functions have to be identical for both the accelerating and decelerating linac passes converging to the same energy and therefore entering the same arc, as illustrated schematically in Fig. 14.

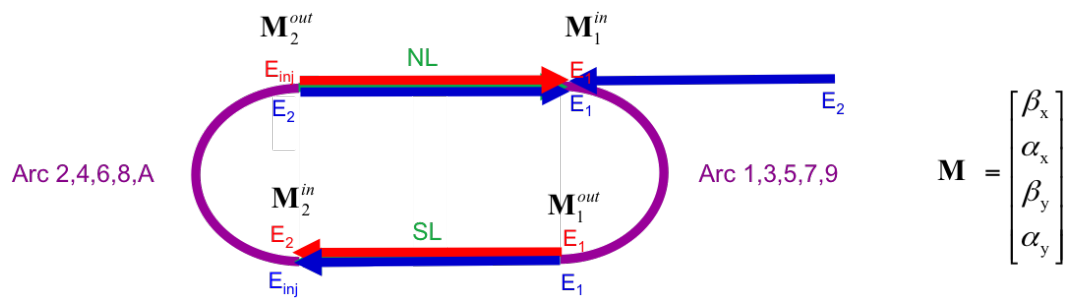

Figure 14: Matching conditions at the linac ends.

To visualize beta functions for multiple accelerating and decelerating passes through a given linac, it is convenient to reverse the linac direction for all decelerating passes and string them together with the interleaved accelerating passes, as illustrated in Fig. 15. This way, the corresponding accelerating and decelerating passes are joined together at the arcs entrance/exit. Therefore, the matching conditions are automatically built into the resulting multi-pass linac beamline. The figure below illustrates the optimum focusing profile for both North and South linacs based on a FODO like ( $60^{\circ}$ phase advance) lattice. One can see that both linacs uniquely define the TWISS functions for the arcs: the NL fixes input to all odd arcs and output to all even arcs, while the SL fixes input to all even arcs and output to all odd arcs.

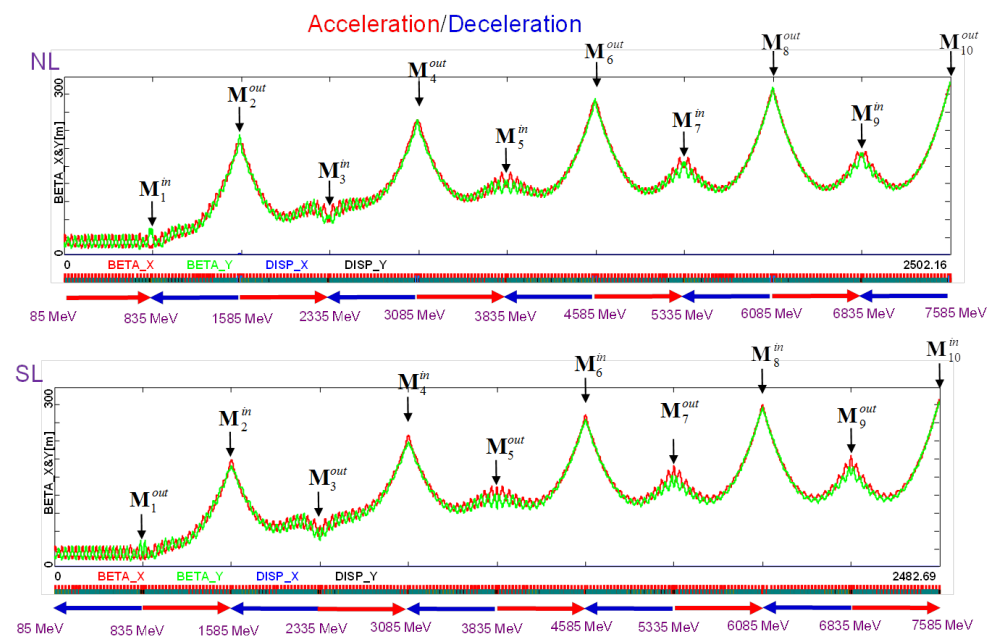

Figure 15: Complete multi-pass optics for both linacs optimized as slightly perturbed $60^{\circ} \mathrm{FODO}$ 
To optimize the multi-pass linac optics, we conducted a thorough exploratory study of optimum phase advance in the linac FODO structure; spanning from no focusing drift linac to a strongly focusing $120^{\circ}$ FODO (present CEBAF linac optics) [29]. A single objective optimization was done to minimize beta/E averaged over all accelerating and decelerating passes; that quantity being a driving term for most collective phenomena in recirculating linacs. As a result, the optimum value of the linac phase advance was found to be $60^{\circ}$. The optimum lowest pass linac optics was configured as a slightly perturbed $60^{\circ}$ FODO (with modified quads at the linac end) — see Fig. 15.

\subsubsection{New Arc Optics for ER}

As we concluded above, both linacs uniquely define the TWISS functions for the arcs. Therefore, the optics settings of all 10 CEBAF arcs will have to be modified to meet the new values of TWISS functions at both arc ends. Since the linacs are almost identical, the required matching conditions are very close to mirror symmetric for the arcs. To redesign the arcs, we modified the spreader and recombiner sections along their matching straights, leaving the arc proper intact. This procedure was carried out for all 10 arcs. The resulting end-to-end 5 passes up +5 passes down optics is illustrated by the piecewise optics in Figs. 16 through 22.

This acceleration process continues pass-by-pass through the SL pass after Arc 9 - see Fig. 19.

Finally, the beam at top energy is transported through Arc A, where it gains an extra half-wavelength (via a path-length delay chicane) and it ends up being decelerated in the following passes through both the North and South linacs as illustrated in Fig. 20.

Similarly, the deceleration continues pass-by-pass through Arc 3, and it finally reaches the last decelerating pass, illustrated in Figs. 21 and 22. Finally, the beam is extracted into the dump at the end of the SL.

Further refinement of this matching is being performed in collaboration with BNL personnel, where the accelerator modeling code Zgoubi is being used to model CEBAF. This code is a field integrator, which is an advantage when modeling and optimizing the (different energy) accelerating and decelerating beams in Arcs 1-9 for ER@CEBAF [30].

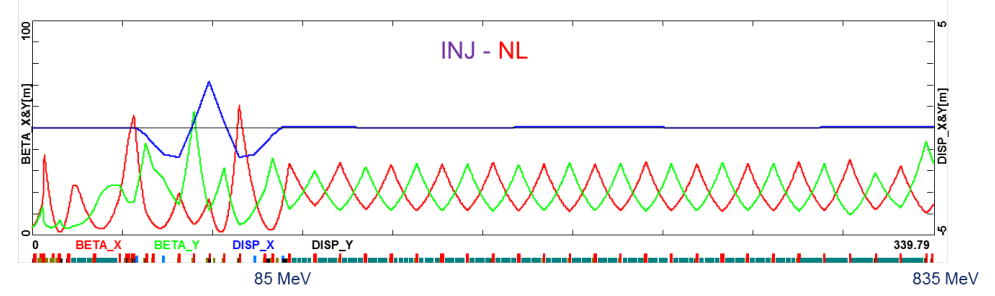

Figure 16: Injector to NL — acceleration.

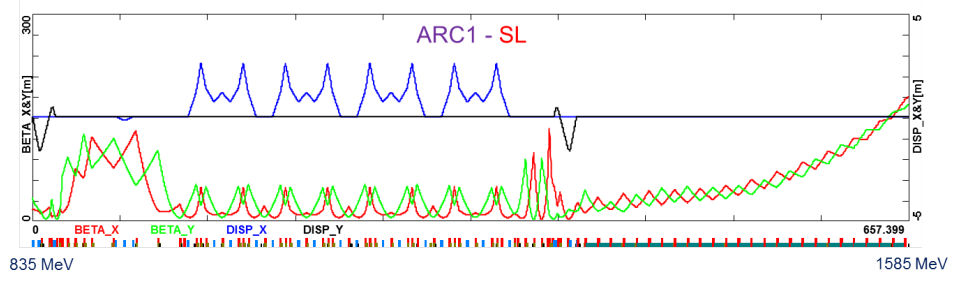

Figure 17: Arc 1 to SL - acceleration. 


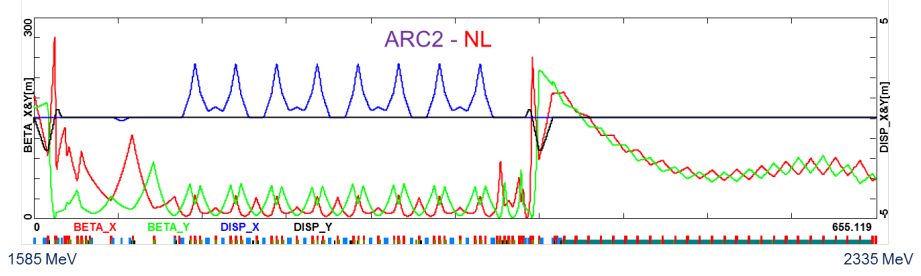

Figure 18: Arc 2 to NL - acceleration.

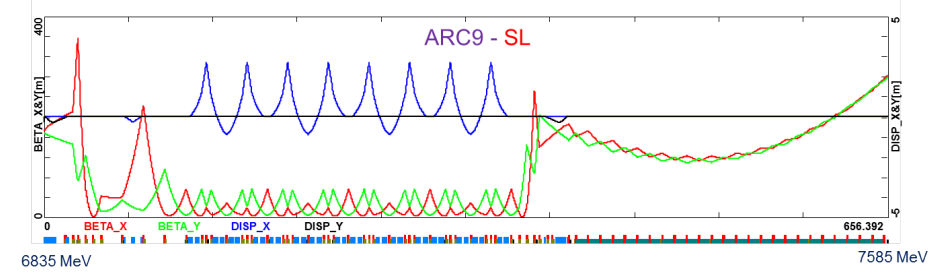

Figure 19: Arc 9 to SL - acceleration.

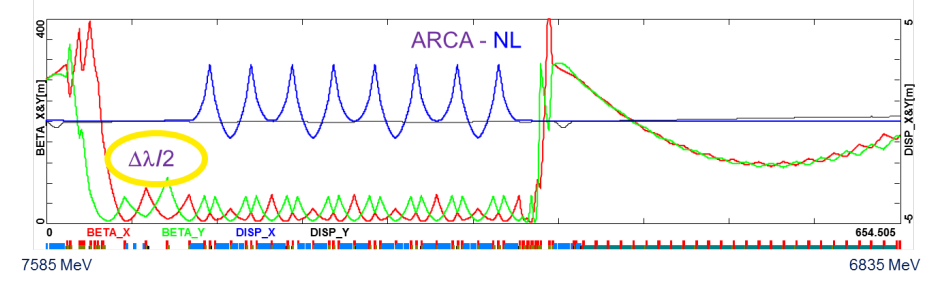

Figure 20: Arc A to NL - deceleration, indicating location of pathlength chicane at start of Arc A.

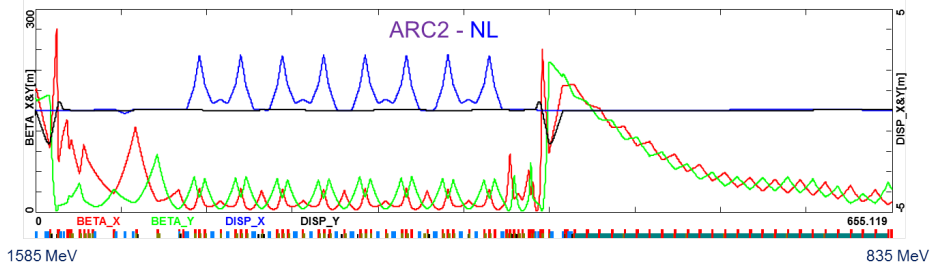

Figure 21: Arc 2 to NL - deceleration.

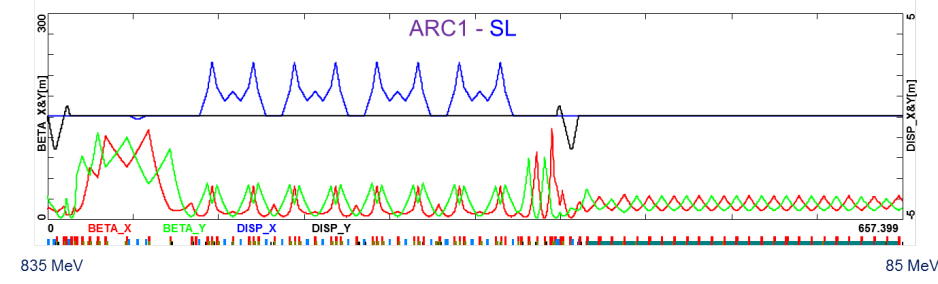

Figure 22: Arc 1 to SL - deceleration. 


\section{Diagnostics and Measurements}

\subsection{Diagnostics}

This section outlines planned diagnostics and measurements for the ER@CEBAF study. Diagnostics are listed with potential calibration needs, coupled with justification from procedural steps. This is likely a minimally sufficient list since a successful demonstration of ER capability will illuminate issues which drive later diagnostic development.

Once the beam is brought to the highest pass, delayed by a half-period of the accelerating RF, and directed into the first decelerating linac, it will undergo an almost unstoppable tumble down the accelerator to its lowest energy and the extraction path. There is no straightforward mechanism with installed beamline components for differential steering control of the accelerating and decelerating passes. The RF separators provide the only obvious way to stop this "avalanche" at intermediate points, simultaneously providing a path for detailed circuit by circuit beam diagnostics on the decelerating path. Preparation may be made as outlined below to divert the beam on selected passes into the Hall-directed extraction lines for characterization.

Setup at two different energies is recommended, the first too low for ISR to make much complication, and the second at an energy for which SR is thought to be a potential limiting factor. SR decreases the beam energy as it accelerates, so that without compensation, the energy of each pass on deceleration is progressively less than for its accelerating counterpart. The momentum acceptance of the recirculation arcs limits the energy discrepancy which can be handled. Accommodation of the momentum mismatch involves detailed longitudinal optics control of the bunch. Measurement of this energy mismatch is included in the proposal.

Multi-energy steering of the beams had been problematic in CEBAF operation without compensation of the skew focusing inherent in the 5 -cell accelerating cavities of the linacs. The cavity RF skew field inverts on deceleration, unlike the DC magnetic compensation. Our accustomed skew compensation is possible only on acceleration or on deceleration, but not both. Recent accelerator tests have demonstrated that with the $12 \mathrm{GeV}$ re-alignment of the linacs, and with linac steering guided by global analysis of the beam trajectories of differing energy, the RF skew focusing no longer poses a large problem. While this compensation is normal operating procedure, it appears that the baseline for this ERL demo should omit skew compensation.

\section{(A) Installation of calibrated terminal diagnostics at Energy Recovery dump}

An energy recovery dump to be installed just downstream from the South Linac will be coupled with wire scanner, CrOx viewer for visual sensitivity, OTR for detailed visual profile measurement, beam current monitor cavity for Machine Protection System (MPS) current conservation monitoring, and a dipole calibrated for total beam energy monitoring. Emittance measurement capability in both planes in the ER dump line is part of the dump line design.

An existing extensive diagnostic complement is installed in the $2 \mathrm{C}$ line that can be used for terminal diagnostics for RF-extracted beams on intermediate passes for comparison of properties between various pass accelerated and decelerated beams. See $(H)$.

Energy from the injector will be precisely measured. Energy gain in the NL will be initially set per the Arc 1 calibration, with principal uncertainty due to geomagnetic contributions. Total energy gain per pass is set by Arc 2 calibration, with parallel uncertainty. The relative energy gains of the NL and SL will be set at the second stage by decelerating the beam in the SL and measuring the residual at the ER dump, adjusting the relative NL/SL energy gain to reproduce the beam energy at the ER dump. Prior to these activities, the cavities of the linacs will be carefully crested to minimize sensitivity to phase drifts.

(B) Instrumental calibration of existing hardware

The time of flight monitor (relative RF phase monitor at $1497 \mathrm{MHz}$, locally called the " $M_{56}$ " system) at the end of the NL must be calibrated for full 360 degree relative phase. This will be validated against beam signals listed in (D).

(C) Extension of CEBAF linac SEE BPMs to 10 passes

The CEBAF linac BPMs use time-of-flight separation of a 4.2 microsecond test pulse in a system with a round-trip circuit time of 4.4 microseconds. During setup in "Tune Mode" each of the 
various pass beam positions is identified by a time delay from a sync pulse. All of the $1497 \mathrm{MHz}$ BPMs fail in measuring concurrent accelerating and decelerating beam pulses because of destructive interference of the two signals. The temporal separation of the accelerating and decelerating beam macropulses allows the linac SEE BPMs to register separately all beam passes. This system is, at this writing, configured only for 5 passes in the South Linac, extended to 6 passes (to support Hall D) in the North Linac. It was extremely useful during the 2003 single-pass ERL demonstration. It will be extended to 10 passes ( 5 up, 5 down) to provide adequate information for setup, operation, and fault detection for the ERL demo.

(D) Procedural beam-based calibration, BPM installation in the $1 / 2$ wave chicane in Arc A:

For validation of (B), the first-pass accelerated beam from the SL will be directly steered along the fifth-pass design beam path into Arc $\mathrm{A}$, with the $1 / 2$ wave chicane set to straight through (zero delay). The chicane will then be set to approximately $1 / 2$ wave delay, for full deceleration of the second pass beam in the NL. Adjustment of the chicane delay so that the decelerated beam energy in Arc 1 is independent of the global "gang phase" of the NL verifies a precise 180 degree delay. Comparison of the energy of the two beams in Arc 1 provides a verification of equality of NL/SL energy gain. BPMs installed in the offset portions of the chicane provide a means to reproduce the 180 degree phase shift for the full-energy beam in Arc A. The injector beam energy will be at least $50 \mathrm{MeV}$, ensuring small non-relativistic corrections to the beam time of flight for acceleration vs. deceleration in the NL.

(E) Installation of selected $3 \mathrm{GHz}$ BPMs and/or OTR foils, minimally 3 locations in each of Arc 1 and Arc 9:

Arc 1 beam energy measurement of the decelerated beam requires knowledge of beam positions in Arc 1. This might be obtained with sufficient accuracy via OTR viewers installed at selected locations in Arc 1, or alternatively by use of $3 \mathrm{GHz}$ BPM signal processing. Another alternative, drilling holes into the existing arc viewers and directing the accelerated beam through the holes, is not as viable a candidate because the full-energy, SR-affected configuration will not necessarily have the accelerated beam at the nominal matched arc energy. A minimal installation would monitor two non-dispersive points and at least one following dispersive point. Installation in Arc 1 supports the beam energy comparisons outlined in (D). A parallel installation in Arc 9 determines the Arc 9 decelerating beam energy. All subsequent beam energies are determined by decisions made to this point, and it is reasonable to verify the result of these decisions in Arc 9. The Arc 1 BPMs also enable a precise comparison of the two beam energies in Arc 1 to test performance of longitudinal manipulations intended to compensate for SR-induced energy droop.

(F) Synchrotron Light Monitors (SLMs) in Arcs 1 and 2, existing diagnostics

Provides relative energy spread comparisons of the two beams in Arc 1 and 2, the accelerating and decelerating beams, in conjunction with other diagnostics.

(G) retrofit earliest $9 \mathrm{~S}$ viewer with a hole to pass accelerating beam

This allows visual detection with a sensitive viewer early in the $9 \mathrm{~S}$ region, supplemented by sensitive current detection with the NL $M_{56}$ system. Reinjection from Arc A along the decelerating path will result in beam falling through 9S to Arc 9 and beyond, with no possibility of an insertable beam stop. Using "viewer-limited," very low duty-factor beam minimizes opportunity for beam spill tripping radiation monitors or RF systems.

(H) Enable RF separation in $8 \mathrm{E} / 6 \mathrm{E} / 4 \mathrm{E} / 2 \mathrm{E}$ selectively to divert decelerating beam into transport extraction lines to carry to Hall B line for detailed phase space diagnostics Low-energy operation permits RF separation on intermediate passes of beam deceleration within available power limits. On next-to-last pass, this is possible in $2 \mathrm{~S}$ always because the acceleration to deceleration phase shift is $1.5 \mathrm{RF}$ wavelengths, rather than the 0.5 waves of $8 \mathrm{E} / 6 \mathrm{E} / 4 \mathrm{E}$. This renders it possible to make detailed phase space measurements on the 4th pass decelerated beam no matter what linac energy gain is selected. Bunch length related head-tail effects from the RF time dependence can increase the measured emittance. However, in $2 \mathrm{E}$, both beams can dwell on peak field of opposite sign, avoiding such emittance dilution. Diverting the beam on its accelerating passes into the extraction lines allows the use of the same diagnostics for the accelerating beam passes 
as the decelerating passes, and also allows for comparison of the properties of static magnetically diverted beam and of RF deflected beam.

There are many detailed steps not included in these descriptions. Careful prioritization and specification of beam parameter measurements is necessary to support selection of diagnostics and comparisons to be made, and to bound systematic uncertainties.

\subsection{Measurements}

In order to avoid head-tail emittance growth, only one RF separator system will be active at any given time. Normal CEBAF operation allows two (or more) RF separators to be active, as convenient for intermediate extraction while beam is directed to higher passes. This is not necessary for the ERL demo, and in principle irrecoverably increases the beam emittance.

A detailed outline of measurements has been generated using the ability to divert beam from its downward cascade into the successive extraction lines. The beam energy for the accelerating and decelerating beams will be measured using the well-calibrated Hall A dipole system ("9th dipole" system), and the 4 meter dispersion optics configuration will allow momentum spread to be measured with IHA1C12 and the associated viewer. At this same time, the machine protection system will provide detailed comparison of the relative current transmission from injector to the accelerated and to the decelerated beam passes. The beam envelope parameters and emittances will be measured in the zero-dispersion $2 \mathrm{C}$ line, instrumented for such measurements. Addition of thicker wires and/or plates to the wire scanner harp will enable high-resolution halo detection on a pass-dependent basis.

Diagnostic opportunity at the final Energy Recovery dump is more restricted than in the $2 \mathrm{C} / 1 \mathrm{C}$ lines, but emittances and momentum spread can also be measured to allow a comparison between an initial NL-accelerated, SL-decelerated beam and the final 5-pass up/down beam with its additional SRdriven energy spread and consequent chromatically driven emittance and halo growth. Dependence of the beam parameters on CW beam current is accessible only for the limited regions presently equipped with synchrotron light monitors. The possibililty of fielding additional such monitors is under consideration, but the Hall A beam line already has such a monitor, well-placed near the high dispersion point, and the ER dump may also be so equipped. 


\section{Cost and Schedule}

\subsection{Installation Cost and Schedule}

Equipment needs for ER@CEBAF are itemized in Table 8, and further sub-itemized by location (extraction linac girder, dump line, and delay chicane). New items are included in costing estimates below. Existing relocated items are relocated within the existing warm area after the SL, and an appropriate beam dump spare exists and can be used for ER@CEBAF.

There is some possibility that two existing MJ $3 \mathrm{~m}$ dipole spares for CEBAF may be used for ER@CEBAF, assuming that permission is obtained to have spares available in the tunnel rather than on the shelf.

Table 8: Itemized Equipment Needs for ER@CEBAF Dump Line and Pathlength Chicane. These include design BDL values for $750 \mathrm{MeV}$ linacs and 5-pass ER (the stretch ER@CEBAF goal).

\begin{tabular}{|c|c|c|c|}
\hline $\begin{array}{l}\text { Section and } \\
\text { Element }\end{array}$ & Usage & Comment & $\begin{array}{l}\text { Design BDL }[\mathrm{G}-\mathrm{cm}] \text { for } \\
750 \mathrm{MeV} \text { linacs }\end{array}$ \\
\hline \multicolumn{4}{|l|}{ Linac Girder } \\
\hline MQL2L27 & Close FODO & Existing Relocated & 9590.382 \\
\hline MBL2L27 & Bend low energy beam into dump line & New & 39389.7 \\
\hline MCA2L27 & Close bump from MBL for high energy beam & New & -39389.7 \\
\hline MDB2L27H & Horizontal correction into spreader & Existing Relocated & 0 \\
\hline MDJ2L27H & Vertical correction into spreader & Existing Relocated & 0 \\
\hline IPM2L27 & Beam position into spreader & Existing Relocated & NA \\
\hline IBC2L27 & Path length cavity & Existing Relocated & NA \\
\hline VDP2L27 & Isolate spreader vacuum & New FEL style & NA \\
\hline \multicolumn{4}{|l|}{ Dump Line } \\
\hline IPMBD00 & Beam position into energy recovery bend & New & NA \\
\hline MBLBD00 & Bend low energy beam to dump line & New & -39389.7 \\
\hline IPMBD00A & Beam position into quad girder & New & NA \\
\hline MQBBD01 & Emittance measurement & New & 14000 \\
\hline MQBBD01A & Emittance measurement & New & 14000 \\
\hline MBCBD01H & Horizontal correction to dump & New & NA \\
\hline MBCBD01V & Vertical correction to dump & New & NA \\
\hline IHABD01 & Beam emittance & New & NA \\
\hline IPMBD01 & Beam position at dump & New & NA \\
\hline IBCBD01 & MPS BCM & New & NA \\
\hline ITVBD01 & Viewer for dump beam & New & NA \\
\hline Beam Dump & Dump energy recovered beam & Exisiting & NA \\
\hline \multicolumn{4}{|c|}{ Delay Chicane } \\
\hline MBAAE00 & Delay chicane 1 & New & 2188004.537 \\
\hline IPMAE00 & Beam position in delay chicane & New & NA \\
\hline MBAAE00 & Delay chicane 2 & New & 4376009.073 \\
\hline MBAAE00A & Delay chicane 3 & New & -2188004.537 \\
\hline MBAAE00B & Delay chicane 4 & New & 2188004.537 \\
\hline
\end{tabular}

All costs for design, procurement, installation, and checkout are included in the overall numbers provided in Table 9. A modest $10 \%$ contingency is recommended as certain cost drivers and escalation cannot be fully predicted. These costs include six new $3 \mathrm{GHz}$ BPMs (described in Section 5), 3 in each of Arcs 1 and 9 to directly measure beam accelerated and decelerated beam energy differences in these areas.

Areas of cost risk are in the large dipole magnets in the chicane as well as the new $3 \mathrm{GHz}$ BPMs. All components have existing designs and the use of spare items is maximized in order to minimize 
procurement of new elements, aside from possible spare MBA 3m dipole use. New vacuum chamber designs are required for the extraction dipole as well as the chicane dipoles. They are based on existing designs and fabrication methods but will be specific for this application.

Table 9: ER@CEBAF Procurement and Installation Costs. These include 10\% management oversight and $10 \%$ contingency in appropriate areas.

\begin{tabular}{|c|c|c|c|c|c|c|c|}
\hline $\begin{array}{l}\text { Design } \\
\text { (weeks) }\end{array}$ & $\begin{array}{l}\text { / Procurement } \\
\text { (FTE) }\end{array}$ & $\begin{array}{l}\text { / QA Labor } \\
(\mathrm{k} \$)\end{array}$ & $\begin{array}{l}\text { Material Cost } \\
(\mathrm{k} \$)\end{array}$ & $\begin{array}{l}\text { Installation / } \\
\text { (weeks) (FTE) }\end{array}$ & $\begin{array}{l}\text { Checkout Labor } \\
(\mathrm{k} \$)\end{array}$ & $\begin{array}{l}\text { Total Cost } \\
(\mathrm{k} \$)\end{array}$ & $\begin{array}{c}\text { Total Labor } \\
\text { (FTE) }\end{array}$ \\
\hline 85.59 & 1.95 & 230.15 & 612.28 & 94.22 & 189.13 & 1031.57 & 4.09 \\
\hline
\end{tabular}

The cost estimate methodology for design and procurements is based on work done as part of the $12 \mathrm{GeV}$ Upgrade to CEBAF. Each item is based on actual costs realized during the build of the $12 \mathrm{GeV}$ accelerator, including magnets, diagnostics, girders, vacuum components, and beamline supports. Labor estimates for installation and checkout are also derived from actual labor data from the $12 \mathrm{GeV}$ Upgrade. FY16 costs were developed by applying a 3\% per annum escalation from the year of the actuals. Labor rates for Jefferson Lab staff are applied for each discrete labor category and reflect FY16 annual work planning values.

An ER@CEBAF installation schedule is shown in Fig. 23. To make the schedule most efficient, the goal is to start early enough such that all procurements and fabrication are completed prior to installation. The design of mechanical items and new vacuum chambers as well as procurement and fabrication of the quantity of $3 \mathrm{GHz}$ BPMs drive the timing. Overall, the schedule is expected to require 6 months of design/procurement/fabrication and another 4 months for installation and checkout. Total project construction and installation schedule is anticipated to be 10 months after access to funding.

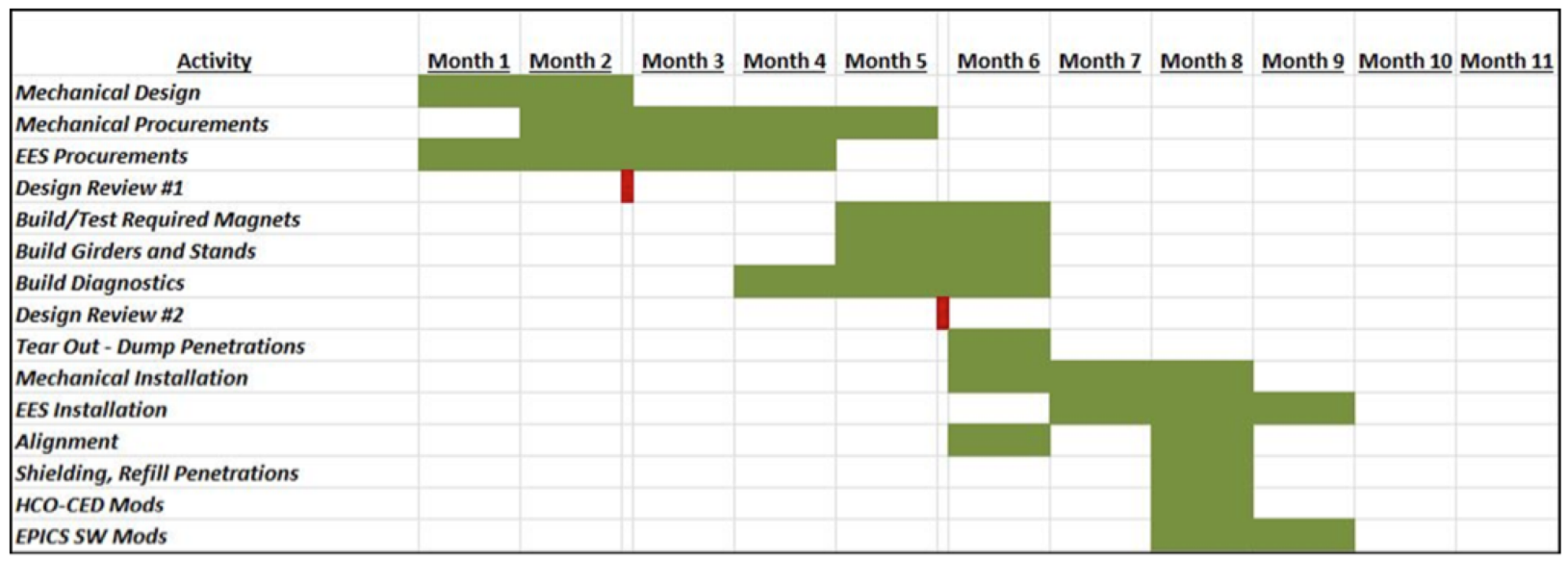

Figure 23: ER@CEBAF installation schedule from start of access to funding.

\subsection{Staged Commissioning Schedule}

Similar to the CEBAF-ER experiment of 2003 [2], we are requesting 12 days of dedicated beam time to commission ER capability in CEBAF after this equipment is installed. This will proceed in three stages:

- Commission dump line/diagnostics (NL acceleration, SL deceleration)

- Commission pathlength chicane and 1-pass ERL

- Commission 5-pass ERL

The first stage should need approximately 1-2 days. This consists of setting the injector energy to $80 \mathrm{MeV}$, lowering the gradients of both linacs to $700 \mathrm{MeV} /$ pass (assuming phasing has already been 
done), rephasing the SL for deceleration, and delivering beam to the dump line for hardware checkout and instrumentation commissioning.

The second stage should need approximately 1 day. The only new hardware is the pathlength chicane, and most of this time is for optics tuning and match with the bypassed quadrupole in the AE region (Fig. 5). Steering of first-pass low-energy beams to the AE region was recently performed in less than one shift for CEBAF $750 \mathrm{MHz}$ separator shunt impedance measurements.

The third stage should take 9-10 days, as it is the bulk of this study and contains nearly all the programmatic uncertainty beyond hardware checkout. This stage requires full spin-up of 5-pass CEBAF in ER conditions ( $700 \mathrm{MeV} /$ linac, new arc optics), a process which is consistently 2-3 days during $12 \mathrm{GeV}$ operations. The remainder of the time is devoted to tuning and characterization of decelerating beam through 5 passes of deceleration, including $\mathrm{CW}$ mode and explorations of energy recovery once 5-pass ER transport has been established.

There are anticipated to be no radiation hazards for this study beyond those found in normal CEBAF $12 \mathrm{GeV}$ operations, as the maximum beam current and energy are within the nominal operations safety envelope. 


\section{References}

[1] N. Sereno, Experimental Studies of Multiplass Beam Breakup and Energy Recovery using the CEBAF Injector Linac, PhD Dissertation, University of Illinois at Urbana-Champaign and Jefferson Lab, 2005.

[2] S.A. Bogacz, A. Hutton et al., "CEBAF-ER Experiment Proposal", Jefferson Lab, PAC22 proposal PR-02-102, June 2002.

[3] C. Tennant, Studies of Energy Recovery Linacs at Jefferson Laboratory, PhD Dissertation, The College of William and Mary and Jefferson Lab, 2006.

[4] G.A. Krafft (ed.) et al., "MEIC Design Summary", arXiv:1504.07961, 29 Apr 2015.

[5] F. Lin et al., "Progress on the Design of the Polarized Medium-energy Electron Ion Collider at JLab", in Proc. of IPAC'15, Richmond, VA, USA, paper TUYB3, pp. 1302-1307.

[6] E.C. Aschenauer et al., "eRHIC Design Study: An Electron-Ion Collider at BNL", arXiv:1409.1633, 18 Dec 2014.

[7] J.L. Abelleira Fernandez et al., "A Large Hadron Electron Collider at CERN: Report on the Physics and Design Concepts for Machine and Detector," Journal of Physics G: Nuclear and Particle Physics 39(7) (2012): 075001.

[8] ERL2015, 56th ICFA Advanced BD Workshop, BNL, June 7-12, 2015, https://www.bnl.gov/ erl2015/ and http://accelconf .web.cern.ch/AccelConf/ERL2015

[9] ICFA Beam Dynamics Newsletter on ERL Technology, No. 68, Eds. J. Wang and W. Chou, Dec. 2015.

[10] L. Merminga, D. Douglas, and G. Krafft, "High-Current Energy-Recovering Electron Linacs", in Annu. Rev. Nucl. Part. Sci. 53, pp. 387-429 (2003).

[11] F. Méot et al., "ER@CEBAF - a High-Energy, Multiple-Pass Energy Recovery Experiment at CEBAF", in Proc. of IPAC'16, Busan, Korea, paper TUOBA02 (contributed oral), May 2016.

[12] A. Freyberger et al., "The CEBAF Energy Recovery Experiment: Update and Future Plans", in Proc. of EPAC'04, paper MOPKF087, pp. 524-6.

[13] C. Tennant, "Energy Recovery Linacs", Chapter 39 of Challenges and Goals for Accelerators in the XXI Century, O. Brüning and S. Meyers eds, World Scientific, 2016.

[14] T. Powers and C. Tennant, "Implications of Incomplete Energy Recovery in SRF-Based Energy Recovery Linacs", in Proc. of ERL'07, Daresbury, UK, p. 75.

[15] R. Kazimi et al., "Observation and Mitigation of Multipass BBU in CEBAF", in Proc. of EPAC'08, Genoa, Italy, paper WEPP087, 2008.

[16] G. Hoffstaetter and I. Bazarov, "Beam-breakup Instability Theory for Energy Recovery Linacs", in Phys. Rev. ST Accel. Beams, 7, p. 054401, 2004.

[17] C. Mayes et al., "The Cornell-BNL FFAG-ERL test accelerator: white paper", arXiv:1504.00588, 2 Apr 2015.

[18] The 2015 Long Range Plan for Nuclear Science, http://science.energy.gov/ /media/np/nsac/ pdf/2015LRP/2015_LRPNS_091815.pdf

[19] V. Ptitsyn et al., "The ERL-based Design of Electron-Hadron Collider eRHIC", in Proc. of IPAC'16, Busan, Korea, paper WEPMW027, 2016.

[20] LHeC Workshop. 24-26 June 2015. CERN (24 June) and Chavannes-de-Bogis (25-26 June), https: //indico.cern.ch/event/356714/

[21] O. Brüning, private communication, CERN, June 2015.

[22] A. Camsonne et al., "A proposal for Compton Electron Detector R\&D", http://wiki.bnl.gov/ conferences/images/0/02/Compton-EIC-Camsonne-June-2014.pdf, 2014.

[23] K.L.F. Bane and P. Emma, "LiTrack: a fast longitudinal phase space tracking code with graphical user interface", in Proc. of PAC'05, Knoxville, TN, paper FPAT091, p. 4266, 2005. 
[24] M. Sands, "The physics of electron storage rings", SLAC-121, SLAC, 1970.

[25] M. Borland, "ELEGANT: A flexible, SDDS-compliant code for accelerator simulation", No. LS-287, Argonne National Laboratory, 2000.

[26] M. Tiefenback, "Beam Performance at JLab/CEBAF, Diagnostics and Metrics", in Proc. of 2012 Beam Instrumentation Workshop, Newport News, VA, paper MOAP01, 2012.

[27] M. Tiefenback, private communication, Jefferson Lab, May 2016.

[28] D. Pellegrini, A. Latina, D. Schulte, and S.A. Bogacz, "Beam-dynamics Driven Design of the LHeC Energy Recovery Linac", in Phys. Rev. ST Accel. Beams, 18, p. 121004, 2015.

[29] S.A. Bogacz, Choice of multi-pass optics for CEBAF-ER, JLAB-Pub TN 15-050 (2015).

[30] ER@CEBAF: Modeling code developments, F. Méot and Y. Roblin, BNL Tech. Note C-A/eRHIC/52 (2016). 